Somat ot opi cal rel at i onshi ps bet ween cort i cal activity and reflex ar eas in refl exol ogy: A functi onal magnet ic resonance i maging st udy

\begin{tabular}{|l|l|}
\hline 著者 & $\begin{array}{l}\text { Nakamar u Tommi , M ur a Naoki , Fukushi ma Ai , } \\
\text { Kawashi ma Ryut a }\end{array}$ \\
\hline $\begin{array}{l}\text { j our nal or } \\
\text { publ i cat i on ti t l e }\end{array}$ & Neur osci ence Let ter s \\
\hline vol une & 448 \\
\hline number & 1 \\
\hline page r ange & $6-9$ \\
\hline year & $2008-12-19$ \\
\hline URL & ht t p: //hdl . handl e. net /10173/353 \\
\hline
\end{tabular}




\title{
Somatotopical relationships between cortical activity and reflex areas in reflexology: a functional magnetic resonance imaging study
}

\author{
Tomomi Nakamaru $^{1,2}$, Naoki Miura ${ }^{3,2}$, Ai Fukushima ${ }^{2}$, Ryuta Kawashima ${ }^{2}$ \\ ${ }^{1}$ Tohoku University School of Medicine, Sendai, Japan. \\ ${ }^{2}$ Department of Functional Brain Imaging, Institute of Development, Aging and Cancer \\ (IDAC), Tohoku University, Sendai, Japan. \\ ${ }^{3}$ Department of Intelligent Mechanical Systems Engineering, Kochi University of Technology, \\ Kami, Japan.
}

Number of pages, figures and Tables: 16 pages / 3 figures / and 1 table.

\#Corresponding Author: Naoki Miura

Department of Intelligent Mechanical Systems Engineering, Kochi University of Technology, 185 Miyanokuchi, Tosayamada-cho, Kami, Kochi, 782-8502 Japan.

TEL: +81-887-57-2314 / FAX: +81-887-57-2013 / E-mail: miura.naoki@kochi-tech.ac.jp

Acknowledgments: This research was supported by JST/RISTEX, R\&D promotion scheme for regional proposals promoted by TAO, and Research center for Language, Brain and Cognition, Graduate school of international cultural studies, Tohoku University. 


\title{
Keywords
}

Functional magnetic resonance imaging, Reflexology, Sensory stimulation, Somatotopy

\begin{abstract}
We examined the somatotopical relationship between cortical activity and sensory stimulation of reflex areas in reflexology using functional magnetic resonance imaging. Three reflex areas on the left foot, relating to the eye, shoulder, and small intestine were stimulated during the experiment. A statistical analysis showed that reflexological stimulation of the foot reflex areas corresponding to the eye, shoulder, and small intestine activated not only the somatosensory areas corresponding to the foot, but also the somatosensory areas corresponding to the eye, shoulder, and small intestine or neighboring body parts. Thus, the findings showed that reflexological stimulation induced a somatosensory process corresponding to the stimulated reflex area and that a neuroimaging approach can be used to examine the basis of reflexology effects.
\end{abstract}




\section{INTRODUCTION}

Reflexology is an alternative medical practice that obtains beneficial effects on the human body by applying pressure to specific points or areas on the feet, hands, and ears, which are called "reflex areas." Each reflex area is believed to correspond to various parts of the human body or organs, and these areas are mapped on the hands and ears and soles of the feet [11]. Reflexology is considered to help eliminate stress, improve blood circulation, and restore the psychological balance of the body. Although this practice has shown positive effects on the human body in some clinical studies $[10,12]$, how reflexology works medically is not yet fully understood. Likewise, the physiological relationships between the reflex areas and the body parts or organs have not yet been investigated.

Recently, fundamental research on alternative medical practices such as acupuncture has progressed using neuroimaging techniques. A previous neuroimaging study suggested that different areas of the somatosensory cortex were activated during stimulation of several acupoints related to different functions [4]. Moreover, Cho et al. reported that part of the visual processing areas was activated when an acupoint related to visual function was stimulated [3]. With regard to reflexology, a previous electroencephalographic (EEG) study investigated the relaxing effects of reflexological treatment [5], but whether sensory stimulation of a reflex area is perceived as stimulation in the corresponding body part by a cognitive process in the 
somatosensory area is unclear.

In this study, we investigated the somatotopical relationship between cortical activity in the somatosensory area and sensory stimulation of reflex areas on the foot using functional magnetic resonance imaging (fMRI). For this purpose, three reflex areas related to the eye, shoulder, and small intestine (SI) were chosen as stimulation points because these areas are separated from each other in the reflexological foot chart and the projection areas of the somatosensory cortex as defined by Penfield's Homunculus [9].

\section{MATERIALS AND METHODS}

Subjects:

Twenty-five right-handed subjects ( 22 men and 3 women aged 18-41 years; mean age 22 years) were enrolled in this study. All participants were healthy (no signs or history of medical or neurological diseases) and were native Japanese speakers. We assessed their handedness based on the Edinburgh Handedness Inventory [8].

Written informed consent was obtained from each participant in accordance with the guidelines of Tohoku University School of Medicine and the Helsinki Final Act of 1975. 
All fMRI measurements were carried out using a 1.5 T Siemens Magnetom Symphony scanner (Siemens, Munich, Germany) at the Research Center for Language, Brain and Cognition, Graduate School of International Cultural Studies, Tohoku University. Slices $(n=33$, slice thickness $=3 \mathrm{~mm}$, gap $=1 \mathrm{~mm}$ ) covering the entire brain were acquired by gradient-echo echo-planner (GE-EPI) MRI [repetition time $(\mathrm{TR})=3000 \mathrm{~ms}$, echo time $(\mathrm{TE})=50 \mathrm{~ms}$, flip angle $=90^{\circ}$, FOV $192 \times 192 \mathrm{~mm}^{2}$, voxel size $=3 \times 3 \times 4 \mathrm{~mm}^{3}$, matrix size $\left.=64 \times 64\right] . \mathrm{In}$ addition, anatomical whole-brain images were obtained by T1-weighted three-dimensional MRI $\left(\mathrm{TR}=1900 \mathrm{~ms}, \mathrm{TE}=3.22 \mathrm{~ms}, \mathrm{FA}=15^{\circ}, \mathrm{FOV}=250 \times 250 \mathrm{~mm}^{2}\right.$, matrix size $=256 \times 256,160$ sagittal slices of $1.25 \mathrm{~mm}$ thickness).

\section{Experimental design:}

Brain activity was measured using a block design consisting of three sensory stimulation tasks of the reflex areas, corresponding to the eye, shoulder, and SI. The duration of each block was $5 \mathrm{~s}$ and the interval between each block was $10 \mathrm{~s}$; each task was repeated 15 times and a 30-s rest period was imposed before the first stimulation. Therefore, the total time of fMRI scanning was $11 \mathrm{~min}, 45 \mathrm{~s}$. The order of tasks was counterbalanced among the subjects. Before the fMRI measurements, an experimenter marked the three reflex areas corresponding to the eye (Fig. 1A), shoulder (Fig. 1B), and SI (Fig. 1C) on the sole of the subject's left foot. The 
experimenter entered the MRI scanner room together with the subject, and the experimenter wore a pair of headphones to receive experimental instructions. In the fMRI environment, the subject was placed in a supine position in the MRI scanner. A semi-lucent screen was put in front of the subject's face, and the subject was instructed to gaze at the fixation-cross on the screen during the fMRI measurement. The experimenter stimulated each reflex area using a wooden stick with the right hand in accordance with the auditory instructions in each block, and touched the subject's left instep with the left hand throughout the experiment.

Data analysis:

Image processing and statistical analysis of the fMRI data were conducted using the statistical parametric mapping software (SPM2; Wellcome Department of Cognitive Neurology, London, UK). The coordinates of all analyses were in Montreal Neurological Institute (MNI) space. The five initial scans for each subject were dummy scans to equilibrate the state of magnetization and were discarded from the time series data. The effect of head motion across the scans was corrected by realigning all scans to the first scan. The slice timing was corrected by adjusting all slices to the sixteenth slice. Then, the data were normalized spatially to the MNI-T1 template, using the T1-weighted anatomical MR image for each subject, and each scan was smoothed with a Gaussian filter in a spatial domain (10 $\mathrm{mm}$ full-width at half -maximum) 
to minimize noise and the effects of normalization errors.

The collected fMRI data were analyzed using a conventional two-stage approach. First, the hemodynamic responses to the different experimental conditions were assessed at each voxel using a general linear model on an intrasubject basis. A hypothesis was made for each intrasubject model in which the hemodynamic responses of the activation fields to the stimulation of the reflex areas corresponding to each eye, shoulder, and SI were assumed to be the canonical hemodynamic response function with a block length of 5-s duration. Global changes were adjusted by proportional scaling, and low-frequency confounding effects were removed using a high-pass filter with a 94-s cutoff. Multiple regression analysis was performed for each voxel to detect the regions in which the MR signal changes were correlated with the hypothesized model. To identify the specific activation associated with stimulation of each reflex area, we generated subtraction images from the parameter estimate of each condition as follows: eye-specific $[($ eye $\times 2)-($ shoulder $+\mathrm{SI})]$, shoulder-specific $[($ shoulder $\times 2)-($ eye + SI)], and SI-specific $[(\mathrm{SI} \times 2)-($ eye + shoulder $)]$ activations. In addition, a subtraction image used to evaluate the common activation for stimulating the sole of the left foot (common: eye + shoulder + SI) was also prepared.

Second, intersubject activation maps were constructed by performing a one-sample t-test on each subtraction image. Since we focused on the relationship between the stimulation 
of a reflex area and the activity of the corresponding somatosensory area, we used a mask image of the postcentral gyrus using the Automatic Anatomical Labeling Atlas provided by WFU_pickatlas [14]. Furthermore, to remove false-positive activation in the statistical testing of each subtraction image, we excluded the voxels that did not reach the level of significance using task versus task contrast (e.g., eye - shoulder) from each statistical test of the subtraction image. We created mask images from activation maps of task versus task contrast, such as "eye shoulder" and "eye - SI" for the statistical test of eye-specific contrast, "shoulder eye" and "shoulder - SI" for the statistical test of shoulder-specific contrast, and "SI - eye" and "SI - shoulder" for the statistical test of SI-specific contrast. The level of significance for each mask image was set at $p<0.05$ (uncorrected). The statistical threshold of each activation map was set at $p<0.05$ [corrected for multiple comparison using family-wise error (FWE) [6] by voxel level]. Finally, the resulting activation maps were constructed and superimposed on a MNI single-subject template.

To evaluate the effect of sensory stimulation of different reflex areas on cognitive processes, a region of interest (ROI) analysis was performed to inspect local changes in the hemodynamic response of the observed activation peaks. We defined a ROI as the peak location of each ROI that appeared when the statistical threshold was set at $p<0.05$ (corrected for multiple comparison using FWE) in the eye-specific, shoulder-specific, and SI-specific 
situations, and the parameter estimates of each condition were extracted. A paired t-test was performed to examine the differences in local signal changes between conditions at each ROI.

\section{RESULTS}

Table 1 shows a summary of the peak locations and t-scores of activated regions in each contrast, and Fig. 3 shows the peak position of activated clusters in each contrast. Activation in the eye-specific contrast was detected in the middle part of the left postcentral gyrus (Fig. 2A). In the case of the shoulder-specific contrast, although no significant activation occurred, the superior part of the right postcentral gyrus tended to show increased local activity (Fig. 2B). Activation in the SI-specific contrast was observed in the superior part of the left postcentral gyrus (Fig. 2C). The superior parts of the right postcentral gyrus (Fig. 2D) and the middle part of the left postcentral gyrus, the same location as that in the eye-specific contrast, were commonly activated during stimulation of the left foot. Fig. 3 shows the parameter estimates of each ROI that was obtained from the eye-specific and SI-specific contrasts.

The parameter estimate under the eye condition was significantly greater than those under the shoulder and SI conditions for the ROI in the middle part of the left postcentral gyrus. In the case of the ROI in the anterior part of the left postcentral gyrus, however, the parameter estimate under the SI condition was significantly greater than those under the eye and shoulder 
conditions.

\section{DISCUSSION}

The purpose of this study was to examine the relationship between cortical activity in the somatosensory area and reflexological stimulation of reflex areas. From the results of the subtraction analysis, sensory stimulation of the reflex areas corresponding to the eye and SI induced different foci of significant activation within the somatosensory area. A sensory nerve for each body part projected on different parts of the somatosensory area, similar to Penfield's Homunculus [1,9]. A previous fMRI study showed that the representation of cortical activity on the somatosensory cortex associated with nociceptive hot and cold sensations in the right hand and foot was consistent with Penfield's Homunculus [2]. Moreover, an EEG study has shown that the areas corresponding to those in Penfield's Homunculus in the primary somatosensory cortex were activated when brief, painful stimuli were applied [7]. Eye-specific activation was observed in the middle part of the postcentral gyrus. The tactile sensation of the face part, including the eye, is affected by the trigeminal nerve, and its signal finally projects to the middle part of the postcentral gyrus. Therefore, sensory stimulation of the reflex area corresponding to the eye was considered to have induced the processing of tactile sensations of the eye or the neighboring area. SI-specific activation was observed in the more superior part of the 
postcentral gyrus. Somatotopical representation of the visceral sensation projects to the very lateral part of the somatosensory cortex, while tactile sensations for the trunk part project to the superior location. A previous fMRI study reported that visceral pain for the intra-abdominal region induced significant activation in the bilateral intra-abdominal region and slight activation in the right trunk region of the somatosensory cortex [13]. Therefore, our results indicate that sensory stimulation of the reflex area corresponding to the SI was related to a tactile sensation of the trunk. In addition, its perception was not necessarily limited to visceral sensation and may include cutaneous sensation in the trunk. Furthermore, these activation clusters from the eye-specific and SI-specific contrasts were located in the left hemisphere, generally meaning that tactile information came from the right side of the body. The theory of reflexology alleges that the reflex area on the left foot reflects the left side of the body. Investigating this inconsistency of laterality will be necessary. The shoulder-specific contrast showed no significant activation, only a tendency for activation in the superior part of the right postcentral gyrus, the location of which was almost consistent with the projection of the tactile sensation to the upper limbs. The difference in the degree of local signal increase under each condition was not clarified in this study. Because this will likely depend on the condition of the subject, it will require more detailed experiments.

Regarding common activation under the various conditions, two significant activated 
clusters were observed: one in the superior part of the right postcentral gyrus, while the other was the same as the eye-specific activation cluster. The former cluster is related to the tactile sensation of the left foot because a tactile sensation from the lower limb projects to the superior part from the medial part of the somatosensory area. Regarding the latter activation, increased local activity under each condition was considered to have affected the contrast of the common condition. From this result, the middle part of the left postcentral gyrus may play a specific role during perceptions of reflexological stimulation of the left foot.

In conclusion, the activated area during the stimulation of each reflex area was consistent with the somatotopic representation of the corresponding or neighboring body parts in the somatosensory area. Previous fMRI studies of acupuncture revealed the somatotopical mapping of acupoints on the forearm, hand, leg and foot [4], and part of the visual processing areas, which were activated when an acupoint related to visual function was stimulated [3]. Those results indicated that reflexology had some effects that were not simply sensory stimulation. Our results support that claim and indicate that a neuroimaging approach may be a useful procedure for examining the underlying effects of this alternative medical practice. 


\section{Acknowledgement}

This research was supported by JST/RISTEX, R\&D promotion scheme for regional proposals promoted by TAO, and Research center for Language, Brain and Cognition, Graduate school of international cultural studies, Tohoku University. 


\section{REFERENCES}

[1] A.K. Afifi, R.A. Bergman, Functional Neuroanatomy: text and atlas second edition. McGraw-Hill Medical Publishing Division, New York, 2005.

[2] H.H. Berman, K.H. Kim, A. Talati, J. Hirsch, Representation of nociceptive stimuli in primary sensory cortex. Neuroreport. 9 (1998) 4179-4187.

[3] Z.H.Cho, S.C.Chung, J.P.Jones, J.B.Park, H.J.Park, H.J.Lee, E.K.Wong, and B.I.Min, New findings of the correlation between acupoints and corresponding brain cortices using functional MRI. Proc. Natl. Acad. Sci. USA 95 (1998) 2670-2673.

[4] A. Nakagoshi, M. Fukunaga, M. Umeda, Y. Mori, T. Higuchi, and C. Tanaka, Somatotopic Representation of Acupoints in Human Primary Somatosensory Cortex: An fMRI study. Magnetic Resonance in Medical Sciences 4 (2005), 187-189.

[5] K. Natarjan, R. Acharya U, F. Alias, T. Tiboleng, S.K. Puthusserypady, Nonlinear analysis of EEG signals at different mental states. BioMedical Engineering OnLine, 3:7 2004.

[6] T.E. Nichols, S. Hayasaka. Controlling the Familywise Error Rate in Functional Neuroimaging: A Comparative Review. Statistical Methods in Medical Research, 12 (2003) 419-446.

[7] Y. Ogino, H. Nemoto, F, Goto, Somatotopy in human primary somatosensory cortex in pain system. Anesthesiology. 103 (2005) 821-827. 
[8] R. Oldfield, The assessment and analysis of handedness: The Edinburgh inventory. Neuropsychologia 9 (1971) 812-815.

[9] W. Penfield, T. Rasmussen, (1968) The Cerebral Cortex of Man: A Clinical Study of Localization of Function. Hafner Publishing Company, New York, 21-25.

[10] I. Siev-Ner, D. Gamus, L. Lerner-Geva, A. Achiron, Reflexology treatment relieves symptoms of multiple sclerosis: a randomized controlled study. Mult Scler 9 (2003) 356-361.

[11] N.L. Stephenson, J.A. Dalton, Using reflexology for pain management. A review. J Holist Nur. 21 (2003) 179-191.

[12] N.L. Stephenson, J.A. Dalton, J. Carlson, The effect of foot reflexology on pain in patients with metastatic cancer. Appl Nurs Res 16 (2003) 284-286.

[13] I.A. Strigo, G.H. Duncan, M. Boivin M. C. Bushnell, Differentiation of Visceral and Cutaneous Pain in the Human Brain, J Neurophysiol 89 (2003) 3294-3303.

[14] N. Tzourio-Mazoyer, B. Landeau, D. Papathanassiou, F. Crivello, O. Etard, N. Delcroix, B. Mazoyer, M. Joliot, Automated anatomical labeling of activations in SPM using a macroscopic anatomical parcellation of the MNI MRI single-subject brain. Neuroimage. 15 (2002) 273-89. 


\section{FIGURE LEGENDS}

Fig. 1. Reflex areas for the (A) eye, (B) shoulder, and (C) small intestine.

Fig. 2. Activated regions in (A) eye-specific, (B) shoulder-specific, (C) SI-specific and (D) common conditions. The upper row shows activated regions projected on the SPM glass brain, and the lower row shows the peak location of each activated region. The activation peak of the left postcentral gyrus under the common condition was the same as the activation peak under the eye-specific condition..

Fig. 3. The mean parameter estimates under each condition at the activated cluster under the (A) eye-specific and (B) SI-specific conditions. The error bar shows the standard error of the mean. The * means that there is a significant difference between two conditions $(\mathrm{p}<0.05)$. 
Figure 1

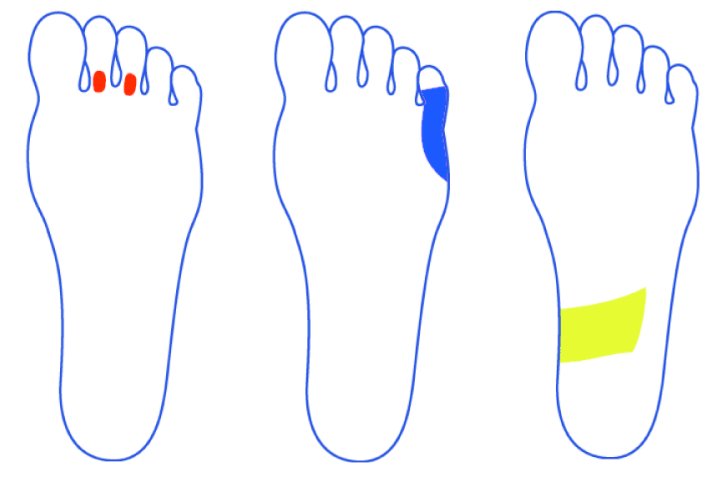

$\begin{array}{lll}\text { (A) Eye } & \text { (B) Shoulder } & \text { (C) Small intestine }\end{array}$ 
Figure 2

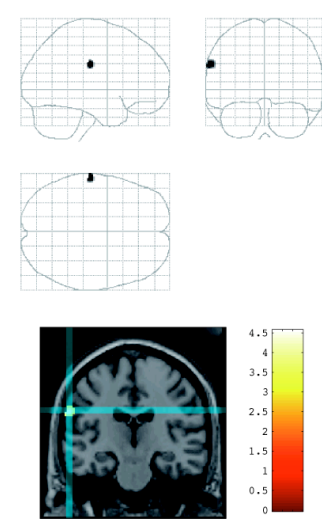

(A) Eye-Specific
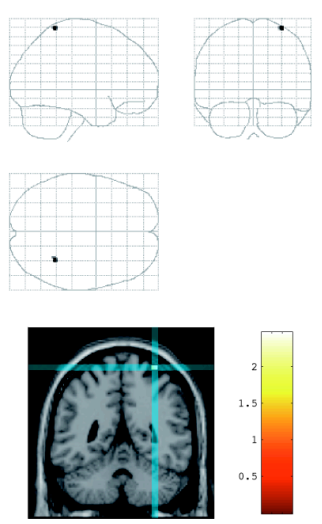

(B) Shoulder-Specific

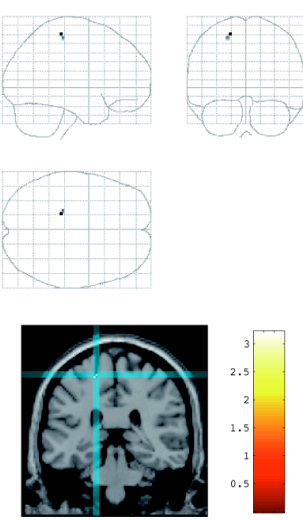

(C) SI-Specific
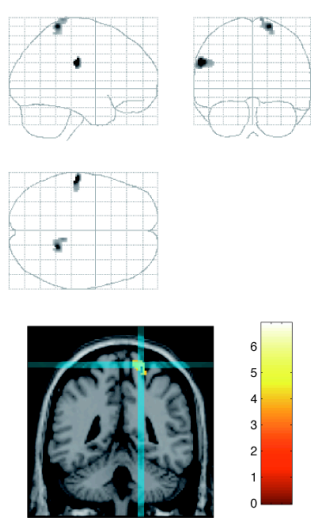

(D) Common 
Figure 3

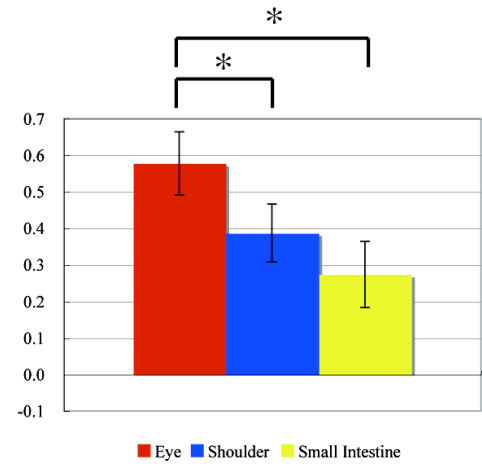

(A) Eye-Specific
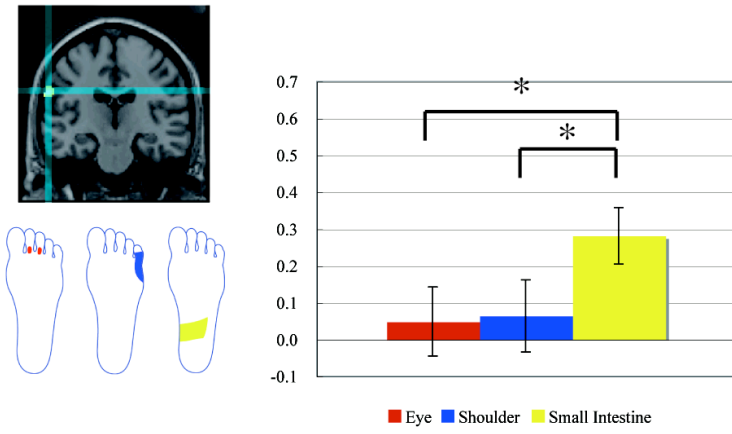

(B) SI-Specific
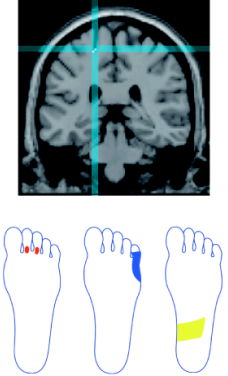

$*: p<0.05$ 